medgen $2017 \cdot 29: 314-315$

https://doi.org/10.1007/s11825-017-0152-4

Online publiziert: 20. Oktober 2017

(c) Der/die Autor(en) 2017. Dieser Artikel ist eine Open-Access-Publikation.

CrossMark

\author{
Verena Steinke-Lange ${ }^{1}$ Stefan Aretz ${ }^{2}$ Evelin Schröck ${ }^{3}$ Elke Holinski-Feder ${ }^{1}$ \\ ${ }^{1}$ Medizinisch Genetisches Zentrum München, München, Deutschland \\ ${ }^{2}$ Institut für Humangenetik, Zentrum für erbliche Tumorerkrankungen, Biomedizinisches Zentrum, \\ Universitätsklinikum Bonn AöR, Bonn, Deutschland \\ ${ }^{3}$ Institut für Klinische Genetik, Medizinische Fakultät, Carl Gustav Carus TU Dresden, Dresden, Deutschland
}

\title{
ERN GENTURIS - Europäisches Referenznetzwerk für erbliche Tumorerkrankungen
}

In der Europäischen Union (EU) sind etwa 30 Mio. Menschen von seltenen Erkrankungen betroffen, welche eine spezialisierte und komplexe Behandlung erfordern. Für viele dieser Erkrankungen gibt es bislang jedoch kein standardisiertes Versorgungskonzept, in strukturschwächeren Ländern fehlt vielen Patienten zudem der Zugang zu einer möglichen Behandlung. Die EU-Kommission hat daher in den letzten Jahren die verbesserte Versorgung dieser Patientengruppe zu einem der Schwerpunkte ihrer Gesundheitspolitik erklärt.

Als eine in diesem Rahmen beschlossene Maßnahme wurde 2016 zur Bildung europaweiter Expertennetzwerke für verschiedene Gruppen seltener oder komplexer Erkrankungen aufgerufen. Insgesamt 24 europäische Referenznetzwerke (ERNs), darunter viele mit deutscher Beteiligung, wurden im März 2017 nach einem aufwendigen Bewerbungs- und Evaluierungsverfahren zugelassen. Sie sollen standardisierte Behandlungskonzepte für ein breites Spektrum seltener Erkrankungen schaffen und allen Patienten grenzübergreifend Zugang zu einer bestmöglichen Versorgung ermöglichen. Zudem soll die Vernetzung auch die Durchführung aussagekräftiger wissenschaftlicher Studien durch Einschluss größerer Patientenzahlen und breiterer fachlicher Expertise ermöglichen.

Das ERN GENTURIS (GENetic TUmor RIsk Syndromes) befasst sich als eines dieser 24 Netzwerke mit der Versorgung von Patienten, die von einem erblichen Tumorsyndrom betroffen sind und somit eine seltene Erkrankung tragen bzw. eine komplexe Versorgung erfordern (www.genturis.eu). Nach derzeitigen Schätzungen werden in Europa nach wie vor 70-80\% dieser Patienten nicht erkannt und ihnen und ihren Angehörigen entsprechend keine adäquaten präventiven Maßnahmen, insbesondere Krebsvorsorge- und Früherkennungsprogramme, angeboten. Tumorerkrankungen werden bei den Patienten und den asymptomatischen Risikopersonen in ihren Familien deshalb häufig erst spät erkannt; syndromspezifische Leitlinien für die Versorgung fehlen zudem häufig.

$\mathrm{Zu}$ den vorrangigen Aufgaben des ERN GENTURIS gehört es deshalb, die Erfassung der betroffenen Familien $\mathrm{zu}$ verbessern und europaweit einheitliche Versorgungskonzepte auf hohem medizinischen Standard zu schaffen. Hierzu sollen interdisziplinäre Behandlungsstandards und Leitlinien geschaffen werden, die über nationale und europaweite Kommunikationsinstrumente und Fortbildungsangebote über die jeweils betreuenden Ärzte allen Patienten zugutekommen. Um die Maßnahmen optimal auf die Bedürfnisse der Betroffenen abzustimmen, werden Patientenvertreter von Anfang an eng in alle Arbeitsbereiche von GENTURIS eingebunden.

Aus Deutschland sind bisher drei klinische Zentren am ERN GENTURIS beteiligt. Hierzu gehören das Institut für Humangenetik des Universitätsklinikums Bonn (Koordination Prof. Stefan Aretz), das Institut für Humangenetik in Dresden (Koordination Prof.
Evelin Schröck) und das Medizinisch Genetische Zentrum in München (Koordination Dr. Verena Steinke-Lange). Die Aufgabe der nationalen Koordinatorin für GENTURIS in Deutschland übernimmt Frau Prof. Elke HolinskiFeder vom Medizinisch Genetischen Zentrum in München. Die einzelnen Zentren koordinieren vor Ort die interdisziplinäre Zusammenarbeit der verschiedenen Fachrichtungen, um eine optimale Versorgung der Patienten mit erblichen Tumorsyndromen zu gewährleisten, und stehen auf nationaler Ebene als Ansprechpartner für andere klinische Zentren, welche Patienten mit erblichem Tumorsyndrom betreuen, zur Verfügung. Die beteiligten Personen engagieren sich auch in verschiedenen Projektgruppen des ERN GENTURIS, welche die zentralen Aufgaben des ERN, wie z.B. die Leitlinienerstellung oder die Einbindung der Patientenvertreter, abdecken sollen.

Das ERN GENTURIS soll somit dazu beitragen, die klinische Versorgung der Patienten mit Tumordispositionssyndromen sowohl auf nationaler Ebene als auch grenzübergreifend zu verbessern und die Kommunikation und Zusammenarbeit der europäischen Experten auf diesem Gebiet zu fördern.

\section{Korrespondenzadresse}

\section{Dr. med. V. Steinke-Lange}

Medizinisch Genetisches Zentrum München Bayerstraße 3-5, 80335 München, Deutschland verena.steinke-lange@mgz-muenchen.de 


\section{Einhaltung ethischer Richtlinien}

Interessenkonflikt. V. Steinke-Lange, S. Aretz, E. Schröck und E. Holinski-Feder geben an, dass kein Interessenkonflikt besteht.

Dieser Beitrag beinhaltet keine von den Autoren durchgeführten Studien an Menschen oder Tieren.

Open Access. Dieser Artikel wird unter der Creative Commons Namensnennung 4.0 International Lizenz (http://creativecommons.org/licenses/by/4.0/deed. de) veröffentlicht, welche die Nutzung, Vervielfältigung, Bearbeitung, Verbreitung und Wiedergabe in jeglichem Medium und Format erlaubt, sofern Sie den/die ursprünglichen Autor(en) und die Quelle ordnungsgemäßnennen, einen Linkzur Creative Commons Lizenz beifügen und angeben, ob Änderungen vorgenommen wurden.

\section{Erste Gentherapie am Auge vor der Zulassung}

Erfolgreiche Therapie eines Enzymmangels

\section{In den USA ist kürzlich erstmals eine Gentherapie gegen Krebs zugelassen worden. Jetzt rechnen deutsche Augenexperten mit einem baldigen Start der ersten Gentherapie auch am Auge.}

Mehr als 250 genetische Sehstörungen sind bekannt, die meisten davon glücklicherweise selten. Ein Beispiel sind Mutationen im Gen RPE65. Es enthält die Information für ein Enzym, das zur Reaktivierung des Sehpigments nach Bleichung benötigt wird - ein Ausfall führt zu einer allmählichen Zerstörung der Sinneszellen in der Netzhaut. Deutschlandweit sind etwa 150 bis 200 Patienten betroffen. Allein 25 kindliche und jugendliche Patienten werden von Professorin Dr. med. Birgit Lorenz an der Justus-Liebig-Universität Gießen betreut. Die Ophthalmologin hofft jetzt auf die baldige Zulassung einer Gentherapie, die in den vergangenen zehn Jahren international an 137 Patienten erprobt worden ist. Bei der Behandlung werden intakte Versionen des RPE65-Gens in Virenhüllen verpackt und über eine Kanüle unter die Netzhaut injiziert.

\section{Eine einmalige Injektion reicht vermutlich aus}

Die bisherigen Ergebnisse sind vielversprechend. "Die Behandlung hat sich in allen publizierten Studien als sicher erwiesen", berichtet Lorenz, operationsbedingte Komplikationen seien sehr selten aufgetreten. Eine einmalige Injektion reicht vermutlich aus, um die Proteinfunktion lebenslang wiederherzustellen. Die Therapie kann abgestorbene Zellen nicht wiederbeleben. Sie kann jedoch das weitere Fortschreiten der Erkrankung verlangsamen.

In den USA hat der Hersteller bereits die Zulassung bei der FDA beantragt; parallel dazu wird der Antrag bei der Europäische Arzneimittel-Agentur EMA vorbereitet. Damit steht die Entwicklung von Gentherapien für angeborene Augenkrankheiten, die vor zehn Jahren begonnen wurde und mittlerweile auch in Deutschland vorangetrieben wird, vor einem ersten Erfolg.

\section{Weiteres Potenzial am Auge}

Eine weitere Gentherapie wird derzeit bei der Chorioideremie erprobt. Ursache sind Mutationen im CHM-Gen auf dem X-Chromosom. Die betroffenen Patienten, alle männ- lich, leiden an einer angeborenen Nachtblindheit und einer Gesichtsfeldeinschränkung, die von außen nach innen fortschreitet. Die Gentherapie zielt darauf ab, die Makula zu erhalten. Bisher wurden 44 Patienten jeweils an einem Auge behandelt, darunter sechs in Tübingen. „Die ersten Langzeitergebnisse sind günstig", berichtet Lorenz. Nach dreieinhalb Jahren hat sich bei zwei von sechs in Oxford behandelten Patienten das Sehvermögen verbessert, während die Erkrankung im unbehandelten Auge weiter fortschritt.

Darüber hinaus werden seit 2015 in Tübingen, München und New York Patienten behandelt, bei denen es aufgrund von $\mathrm{Mu}$ tationen im Gen CNGA3 zum Ausfall der Zapfen kommt, die in der Netzhaut für das Farbensehen zuständig sind. Die Folge ist Achromatopsie, eine extreme Farbenblindheit. „Da die Stäbchen für das Schwarzweißsehen im Hellen nicht funktionieren sind die Patienten bei Tag quasi blind, wenn sie nicht die Augen zukneifen, um möglichst wenig Licht in die Augen fallen zu lassen", beschreibt Lorenz das Krankheitsbild. Auch hier könnte eine Gentherapie helfen, die Zapfen wieder funktionstüchtig zu machen. Die ersten Ergebnisse der Studie, die in Deutschland initiiert wurde, werden für das Frühjahr 2018 erwartet.

\section{Verbesserung der Gentherapie durch Genom-Editoren}

In Gießen wird derzeit an einer grundsätzlichen Verbesserung der Gentherapie gearbeitet. „Statt mit Genfähren zusätzliche DNA in die Zellen zu schleusen, sollen moderne Genom-Editoren die mutierten Gene gleich vor Ort reparieren", erklärt Professor Lorenz. Ob dies gelingt, lässt sich derzeit nicht absehen. Die Experimente befinden sich noch im Laborstadium.

\section{Kerstin Ullrich, Pressestelle der DOG} http://www.dog.org 